Karl-Franzens Universität Graz

Technische Universität Graz

Medizinische Universität Graz

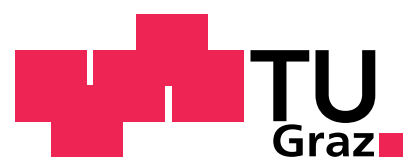

\title{
Boundary control of bidomain equations with state dependent switching source functions in the ionic model
}
N. Chamakuri
C. Engwer
K. Kunisch 
SFB sponsors:

- Austrian Science Fund (FWF)

- University of Graz

- Graz University of Technology

- Medical University of Graz

- Government of Styria

- City of Graz

Das Land

Steiermark

Stadt $\mathbf{G} \mathbf{R}$ A Z $\mathbf{Z}$ Wissenschaft 


\title{
Boundary control of bidomain equations with state dependent switching source functions in the ionic model
}

\author{
Nagaiah Chamakuri ${ }^{1 *}$, Christian Engwer ${ }^{2 \dagger}$, and Karl Kunisch ${ }^{1,3 \ddagger}$ \\ ${ }^{1}$ Radon Institute for Computational and Applied Mathematics, \\ Linz, Austria. \\ ${ }^{2}$ Fachbereich Mathematik und Informatik der \\ Universität Münster, Germany. \\ ${ }^{3}$ Institute of Mathematics and Scientific Computing, \\ University of Graz, Austria.
}

November 4, 2013

\begin{abstract}
Optimal control for cardiac electrophysiology based on the bidomain equations in conjunction with the Fenton-Karma ionic model is considered. This generic ventricular model approximates well the restitution properties and spiral wave behavior of more complex ionic models of cardiac action potentials. However, it is challenging due to the appearance of state-dependent discontinuities in the source terms. A computational framework for the numerical realization of optimal control problems is presented. Essential ingredients are a shape calculus based treatment of the sensitivities of the discontinuous source terms and a marching cubes algorithm to track iso-surface of excitation wave fronts. Numerical results exhibit successful defibrillation by applying an optimally controlled extracellular stimulus.
\end{abstract}

Keywords: bidomain model, Fenton-Karma ionic model, defibrillation, Neumann boundary stimulation, optimal control, state dependent discontinuities.

\section{Introduction}

While the electrical activation of the heart is highly organized in healthy situations, in the diseased case disturbances in the formation and/or propagation of electrical impulses may induce arrhythmias which precipitate its rhythm significantly. Such fast rhythms may evolve to highly disorganized activation patterns. Their termination can be achieved by applying a strong electrical shock, a process called defibrillation. The link between the high shock strengths required and adverse effects provides the motivation for posing the defibrillation process as an optimization problem where one aims to achieve defibrillation with minimal energy.

The optimal control approach to defibrillation is to determine an applied electrical field in such a way that it optimizes a given design objective, which is, in our case, the restoration of a tissue state in which fibrillating propagation cannot be maintained. This can be achieved by driving the whole tissue to a resting state, or alternatively, to an excited state from where the system reaches the equilibrium without further stimuli. In both cases the goal consists in diminishing the main ingredients for maintaining fibrillation, namely

\footnotetext{
*nagaiah. chamakuri@ricam. oeaw.ac .at

$\dagger$ christian. engwer@uni-muenster.de

${ }^{\ddagger}$ karl . kunisch@uni-graz.at
} 
the presence of both propagating wavefronts and a sufficient mass of excitable tissue at rest, in which these wavefronts can travel.

Achieving these objectives is challenging since, on biophysical grounds, shock-induced changes in polarization of both polarities are always present during shock delivery $[28,26]$.

In previous work we addressed this problem for the phenomenological FitzHughNagumo and the physiological Luo Rudy models, with control action modeled either as a distributed controller acting on a small tissue patch or as an inhomogeneous Neumann boundary condition. Here the focus lies on understanding the more complex phenomenological Fenton Karma model [17] in the context of numerical optimal control. It can be considered as a simplification of the Luo-Rudy 1 model of the cardiac membrane that reproduces quantitatively much of the behavior of the full model. This model contains three currents, loosely corresponding to sodium, calcium, and potassium. It preserves important properties of cardiac tissue including action potential rate of rise, different time scales for depolarization and repolarization, action potential duration and conduction velocity restitution curves, and nonzero minimum diastolic interval. Compared to the FitzHughNagumo model it does not have a Maxwell point, and thus its dynamics are comparable to complex ionic models as they have a well-defined minimum diastolic interval and minimum action potential duration.

From the numerical and optimal control points of view the model is challenging due to the appearance of a state-dependent Heaviside function. The effect of the discontinuous source functions appearing in the ionic model equations can be increased by virtual electrodes [28]. This may lead to complex evolutions of the interfaces and constitutes a serious numerical challenge. In the cardiac electrophysiological modeling these difficulties arise not only for the Fenton Karma model but for other models like the Mitchel Schäffer, van Capelle-Durrer or the ten Tusscher model as well, see e.g. [21, 34, 33]. Concerning the bidomain model equations themselves there are several survey articles from among which we quote $[18,27,32]$.

We shall meet these difficulties by applying an extended marching cubes algorithm to track the moving interfaces $[16,3]$ and by using methods from shape optimization to formally derive the sensitivity and adjoint equations for optimal control. The extended marching-cubes algorithm allows the efficient computation of the interface, with additional topological guarantees. To evaluate integrals over the interfaces we impose a piecewise linear reconstruction of the level sets, which is sufficient for first-order representations of the state variable. Using the dune-mc library [16] we compute this polygonal reconstruction of the linearized iso-surface on each cell. The code supports computations in 2D and 3D, thus the current $2 \mathrm{D}$ simulations can directly be extended to the $3 \mathrm{D}$ case.

The organization of the paper is as follows: The model equations describing the dynamical behavior in terms of the extracellular potential, the transmembrane voltage, and the gating variables based on the Fenton Karma ionic model are presented in the following section. The optimal control formulation and the derivation of the optimality system are given in Section 3. It requires to take variations of switching functions with respect to the transmembrane voltage, which is the state variable of the parabolic equation. This is addressed in Section 4 where we make use of the speed method, which is well known in the theory of shape optimization. The spatial and temporal discretizations of the state equations as well as the complete optimality system are presented in Section 5. Numerical results for the termination of reentry waves by using the approximation of the derivatives of Heaviside source functions, which arise in the dual equations due to the specific Fenton-Karma ionic model, are shown in Section 6. 


\section{Governing equations}

Let $\Omega \subset \mathbb{R}^{d}, d \in\{2,3\}$, denote a bounded connected domain with Lipschitz continuous boundary $\partial \Omega$. The space-time domain and its lateral boundary are denoted by $Q=\Omega \times$ $(0, T]$ and $\Sigma=\partial \Omega \times(0, T]$, respectively. The well known and complete mathematical model for describing the electrical activity of the heart are the bidomain equations [18, 27, 32]. Mathematically, they consist of a linear elliptic partial differential equation and a nonlinear parabolic differential equation of reaction-diffusion type, where the reaction term is described by a set of ordinary differential equations. The dimension of the ODE system is determined by the ionic model.

Here we use the Fenton-Karma model [17] to describe the membrane ionic activity. It is derived based on two well known physiological models, the Luo-Rudy [20] and the BeelerReuter [5] models. The Fenton-Karma model consists of three ionic currents, $I_{f i}(v, f)$, $I_{s i}(v, s)$ and $I_{s o}(v)$ which represent the flows of sodium, calcium and potassium. They are accurate enough to produce the membrane recovery processes that give rise to generic restitution curves. The fast inward current $I_{f i}(v, f)$ is responsible for depolarization of the membrane. This depends on transmembrane voltage and on one inactivation-reactivation gate $f$. The main activity of this gating variable is for inactivation of the current after depolarization of the cardiac tissue and also its reactivation after repolarization. The slow outward current $I_{s o}(v)$ which is responsible for repolarization of the membrane depends only on the transmembrane voltage. The slow inward current $I_{s i}(v, s)$ balances the slow outward current during the plateau phase of the action potential. It mainly depends on the transmembrane voltage and one gating variable $s$ which is responsible for inactivation and reactivation of the current. The complete bidomain equations together with the ODE system are expressed as follows.

$$
\begin{aligned}
0 & =\nabla \cdot\left(\bar{\sigma}_{i}+\bar{\sigma}_{e}\right) \nabla u+\nabla \cdot \bar{\sigma}_{i} \nabla v \quad \text { in } Q \\
\frac{\partial v}{\partial t} & =\nabla \cdot \overline{\sigma_{i}} \nabla v+\nabla \cdot \bar{\sigma}_{i} \nabla u-\left(I_{f i}(v, f)+I_{s i}(v, s)+I_{s o}(v)-I_{s t i m}\right) \text { in } Q \\
I_{f i} & =-f g(v)\left(v-v_{c}\right)(1-v) / \tau_{f i} \text { in } Q \\
\frac{\partial f}{\partial t} & =(1-g(v)) \frac{(1-f)}{\tau_{f}^{-}(v)}-\frac{g(v) f}{\tau_{f}^{+}} \text {in } Q, \\
I_{s i} & =-s\left(1+\tanh \left(\kappa\left(v-v_{c}^{s i}\right)\right)\right) /\left(2 \tau_{s i}\right) \quad \text { in } Q \\
\frac{\partial s}{\partial t} & =(1-g(v)) \frac{(1-s)}{\tau_{s}^{-}}-\frac{g(v) s}{\tau_{s}^{+}} \text {in } Q, \\
I_{s o} & =v(1-g(v)) / \tau_{0}+g(v) / \tau_{r} \quad \text { in } Q,
\end{aligned}
$$

where $u: Q \rightarrow \mathbb{R}$ is the extracellular potential, $v: Q \rightarrow \mathbb{R}$ is the transmembrane voltage, $f$ and $s: Q \rightarrow \mathbb{R}$ represent the ionic current variables, $\bar{\sigma}_{e}, \bar{\sigma}_{i}: \Omega \rightarrow \mathbb{R}^{d \times d}$ are respectively the extra and intracellular conductivity tensors, $I_{f i}$ is the fast inward current, $I_{s i}$ is the slow inward current and $I_{s o}$ is the slow (ungated) outward current. Eq. (2) is a parabolic equation and Eq. (4-6) are a set of ordinary differential equations with time and space dependent state variables $f$ and $s$.

Most importantly, the complete behavior of the gating variables and the dynamics of transmembrane voltage is characterized by the step function

$$
g(v)= \begin{cases}1 & \text { if } v \geq v_{c} \\ 0 & \text { if } v<v_{c}\end{cases}
$$

where $v_{c}$ is the critical value of transmembrane voltage. The function $\tau_{f}^{-}$in the fast gating equation is defined by

$$
\tau_{f}^{-}(v)=(1-g(v)) \tau_{f 1}^{-}+g(v) \tau_{f 2}^{-} .
$$


The appearance of $g$ in the physiological model poses analytical as well as numerical challenges. For the optimal control approach we require the sensitivity equations and hence the first order variations of $g$ with respect to $v$. The numerical realization of the wave fronts will be accomplished by the aforementioned marching cube algorithm.

The distribution of conductivity tensors considered of paramount importance and can lead to suprathreshold depolarization during a defibrillation shock [29, 1]. It may lead to the establishment of regions of membrane depolarization and hyperpolarization and eventually lead to a successful defibrillation. In general the conductivity tensor is symmetric positive definite. In our computations, the conductivity tensors are of the following form,

$$
\sigma_{c}=\left(\begin{array}{cc}
\sigma_{c l} & 0 \\
0 & \sigma_{c t}
\end{array}\right), \quad \text { where } c=i, e,
$$

and $\sigma_{c l}$ and $\sigma_{c t}$ are the longitudinal and transverse fiber conductivities, respectively. In our work the heterogeneity is drawn from the histological image of the cardiac tissue as will be explained in Section 6 .

The initial and boundary conditions are prescribed by

$$
\begin{aligned}
\mathbf{n} \cdot\left(\sigma_{i} \nabla v+\sigma_{i} \nabla u\right)= & 0 \text { on } \Sigma \\
\mathbf{n} \cdot \sigma_{e} \nabla u= & I_{e} \text { on } \partial \Omega_{12} \times(0, T] \\
\mathbf{n} \cdot \sigma_{e} \nabla u= & 0 \text { on } \partial \Omega_{3} \times(0, T] \\
v(x, 0)=v_{0}, & f(x, 0)=f_{0}, \quad \text { and } s(x, 0)=s_{0} \quad \text { on } \Omega,
\end{aligned}
$$

where $\mathbf{n}$ denotes the outwards normal to the boundary of $\Omega$. Here $I_{e}$ is the extracellular current density stimulus which acts as control along the boundary $\partial \Omega_{12}=\partial \Omega_{1} \cup \partial \Omega_{2}$, where $\partial \Omega_{i}, i=1,2,3$ are mutually disjoint and satisfy $\partial \Omega_{1} \cup \partial \Omega_{2} \cup \partial \Omega_{3}=\partial \Omega$. For compatibility reasons it is assumed throughout that

$$
\int_{\partial \Omega} I_{e}(t, \cdot) d s=0
$$

for almost every $t \in(0, T)$. In the numerical experiments $I_{e}$ will be only temporally dependent and will be of the form

$$
I_{e}=\hat{I}_{e}(t)\left(\chi_{\partial \Omega_{1}}-\chi_{\partial \Omega_{2}}\right),
$$

where $\chi_{\partial \Omega_{i}}$ is the characteristic function of the set $\partial \Omega_{i}, i=1,2$. Then condition (14) is satisfied if $\left|\partial \Omega_{1}\right|=\left|\partial \Omega_{2}\right|$. The support regions $\partial \Omega_{1}$ and $\partial \Omega_{2}$ can be considered to represent a cathode and an anode, respectively.

\section{Optimal control formulation}

We describe the optimal control formulation for defibrillation of undesired arrhythmias. It involves tracking to a desired state $v_{\alpha}$ which is known to lead to defibrillation, as well as a penalization of the extracellular current stimulus $I_{e}$ which serves as a control and in practice has an averse effect on the tissue. The optimal control problem then is of the form

$$
\left\{\begin{array}{l}
\min J\left(v, I_{e}\right)=\frac{1}{2} \int_{0}^{T}\left(\alpha_{1} \int_{\Omega_{o b s}}\left|v-v_{d}\right|^{2} d x+\alpha_{2} \int_{\partial \Omega_{12}} I_{e}(t)^{2} d s\right) d t \\
\text { subject to (1)-(6),(10)-(13) and } I_{e} \in U,
\end{array}\right.
$$

where $\alpha_{1}>0, \alpha_{2}>0$ are the regularization parameters $\Omega_{o b s} \subset \Omega$ is the observation domain, $v_{d} \in L^{2}\left(0, T ; L^{2}\left(\Omega_{o b s}\right)\right)$ and

$$
\begin{array}{r}
U=\left\{I_{e}-\frac{1}{\left|\partial \Omega_{12}\right|} \int_{\partial \Omega_{12}} I_{e} d s: I_{e} \in L^{2}\left(0, T ; L^{2}\left(\partial \Omega_{12}\right)\right),\left|I_{e}(t, x)\right| \leq R\right. \\
\text { for a.e. } \left.(t, x) \in(0, T) \times \partial \Omega_{12}\right\} .
\end{array}
$$


Here we proceed formally and assume the well posedness of problem (15). At a solution $I_{e}^{\star}$ the first order necessary condition must be satisfied. To establish this condition we introduce the Lagrangian in the following form:

$$
\begin{aligned}
\mathcal{L}\left(u, v, f, s, I_{e}, p, q, r, o\right)=J\left(v, I_{e}\right) & \\
& +\int_{0}^{T} \int_{\Omega}\left(\nabla \cdot\left(\bar{\sigma}_{i}+\bar{\sigma}_{e}\right) \nabla u+\nabla \cdot \bar{\sigma}_{i} \nabla v\right) p \mathrm{~d} \Omega \mathrm{d} t \\
& +\int_{0}^{T} \int_{\Omega}\left(\nabla \cdot \bar{\sigma}_{i} \nabla v+\nabla \cdot \bar{\sigma}_{i} \nabla u-\frac{\partial v}{\partial t}-\left(I_{f i}+I_{s i}+I_{s o}\right)\right) q \mathrm{~d} \Omega \mathrm{d} t \\
& +\int_{0}^{T} \int_{\Omega}\left(\frac{(1-g)(1-f)}{\tau_{f}^{-}(v)}-\frac{g f}{\tau_{f}^{+}}-\frac{\partial f}{\partial t}\right) r \mathrm{~d} \Omega \mathrm{d} t \\
& +\int_{0}^{T} \int_{\Omega}\left(\frac{(1-g)(1-s)}{\tau_{s}^{-}}-\frac{g s}{\tau_{s}^{+}}-\frac{\partial s}{\partial t}\right) o \mathrm{~d} \Omega \mathrm{d} t \\
& +\int_{0}^{T} \int_{\partial \Omega_{12}}\left(\bar{\sigma}_{e} u-I_{e}\right) p^{N} \mathrm{~d} \Omega \mathrm{d} t
\end{aligned}
$$

where the initial and boundary conditions are kept as explicit constraints. The first order optimality system is obtained by formally setting the partial derivatives of $\mathcal{L}$ equal to 0 . Taking the derivative of $\mathcal{L}$ w.r.t. the state variables $u, v, f$ and $s$ gives the following adjoint equations for the adjoint state variables $p, q, r$ and $o$ respectively.

\section{Adjoint equations}

$$
\begin{aligned}
0 & =\nabla \cdot\left(\bar{\sigma}_{i}+\bar{\sigma}_{e}\right) \nabla p+\nabla \cdot \bar{\sigma}_{i} \nabla q \text { in } Q \\
\frac{\partial q}{\partial t} & =-\nabla \cdot \bar{\sigma}_{i} \nabla p-\nabla \cdot \bar{\sigma}_{i} \nabla q+I_{v}(v, f, s) q+F_{v}(v, f) r+S_{v}(v, s) o \text { in } Q \\
\frac{\partial r}{\partial t} & =-F_{f}(v, f) r+I_{f}(v, f, s) q \text { in } Q, \\
\frac{\partial o}{\partial t} & =-S_{s}(v, s) o+I_{s}(v, f, s) q \text { in } Q,
\end{aligned}
$$

where the subscripts $v, f, s$ denote the partial derivatives w.r.t. these variables and $F(v, f)$ and $S(v, s)$ are the right hand side of (4) and (6) respectively. The expressions for the partial derivatives are provided below. First, the derivative of the ionic current w.r.t. the transmembrane voltage is given by the following expressions

$$
\begin{aligned}
I_{v}(v, f, s) & =\left(I_{f i}\right)_{v}+\left(I_{s i}\right)_{v}+\left(I_{s o}\right)_{v}, \text { where } \\
\left(I_{f i}\right)_{v} & =-\frac{f g}{\tau_{f i}}\left[-\left(v-v_{c}\right)+(1-v)\right]-\frac{f\left(v-v_{c}\right)(1-v)}{\tau_{f i}} d g(v) \\
\left(I_{s i}\right)_{v} & =-\frac{s}{2 \tau_{s i}}\left[\left(1-\tanh ^{2}\left(\kappa\left(v-v_{c}^{s i}\right)\right) \cdot \kappa\right]\right. \\
\left(I_{s o}\right)_{v} & =\frac{1-g}{\tau_{0}}+\left(\frac{-v}{\tau_{0}}+\frac{1}{\tau_{r}}\right) d g(v)
\end{aligned}
$$

and for the fast and slow gating variables we obtain:

$$
\left(I_{f i}\right)_{f}=-\frac{g\left(v-v_{c}\right)(1-v)}{\tau_{f i}}, \quad\left(I_{s i}\right)_{s}=-\left[1+\tanh \left(\kappa\left(v-v_{c}^{s i}\right)\right)\right] /\left(2 \tau_{s i}\right) .
$$


The partial derivative of the right hand side of the gating variables are:

$$
\begin{aligned}
F(v, f) & =\frac{(1-g)(1-f)}{\tau_{f}^{-}(v)}-\frac{g f}{\tau_{f}^{+}}, \quad S(v, s)=\frac{(1-g)(1-s)}{\tau_{s}^{-}}-\frac{g s}{\tau_{s}^{+}} \\
F_{f}(v, f) & =-\frac{1-g}{\tau_{f}^{-}(v)}-\frac{g}{\tau_{f}^{+}}, \quad S_{s}(v, f)=-\frac{1-g}{\tau_{s}^{-}}-\frac{g}{\tau_{s}^{+}} \\
F_{v}(v, f) & =\frac{-\tau_{f}^{-}(v)[(1-f) d g(v)]-[1-g(v)](1-f)\left(\tau_{f}^{-}(v)\right)_{v}}{\left[\tau_{f}^{-}(v)\right]^{2}}-\frac{d g(v)}{\tau_{f}^{+}} \\
S_{v}(v, s) & =-\frac{(1-s) d g(v)}{\tau_{s}^{-}}-\frac{s d g(v)}{\tau_{s}^{+}}, \quad\left(\tau_{f}^{-}(v)\right)_{v}=-d g(v) \tau_{v_{1}}^{-}+d g(v) \tau_{v_{2}}^{-}
\end{aligned}
$$

Here we need to evaluate the variation of $v \rightarrow g(v)$ to obtain $d g(v)$. The evaluation of such derivatives is addressed in the following section.

The terminal conditions of the dual equations are defined as follows

$$
q(T)=0, r(T)=0, o(T)=0
$$

and the boundary conditions for the adjoint states are

$$
\begin{aligned}
\mathbf{n} \cdot\left(\bar{\sigma}_{i} \nabla q+\bar{\sigma}_{i} \nabla p\right) & =0 & & \text { on } \Sigma, \\
\mathbf{n} \cdot \bar{\sigma}_{e} \nabla p & =0 & & \text { on } \Sigma,
\end{aligned}
$$

and $\int_{\Omega} p(t) d x=0$, for a.e. $t \in(0, T)$.

Moreover, for any optimal control $I_{e}^{*}$ the following variational inequality must be satisfied:

$$
\int_{0}^{T} \int_{\partial \Omega_{12}}\left(\alpha I_{e}^{*}+Q p\right)\left(I_{e}-I_{e}^{*}\right) d s d t \geq 0, \text { for all } I_{e} \in U
$$

where $(Q p)(t)=p(t)-\frac{1}{\left|\partial \Omega_{12}\right|} \int_{\partial \Omega_{12}} p(t, s) d s$ on $\partial \Omega_{12}$.

\section{Approximation of derivatives of discontinuous source func- tions}

In this section we describe our approach to approximate the derivative of discontinuous source functions. To compute the gradient of the Lagrangian $\mathcal{L}$ with respect to $v$ we need to evaluate the gradient of functionals of the form

$$
j(v)=\int_{\Omega} g(v(x)) f(x) d x,
$$

where $g(v(x))=1$ if $v(x)>v_{c}$ and $g=0$ otherwise. The lack of differentiability of $g$ would suggest the use of level set techniques or regularization procedures, see e.g. [30, 15]. For the latter the choice of a proper regularization parameter is crucial and, as pointed out in [15], standard delta discretizations can diminish convergence. Here we proceed along a different route and take advantage of shape analysis techniques.

For this purpose let $h(x)$ be a function defined on $\Omega$ and consider the derivative of $\frac{d}{d v} j(v)$ in direction of $h(x)$ as

$$
\lim _{t \rightarrow 0^{+}} \frac{1}{t}(j(v+t h)-j(v))=\lim _{t \rightarrow 0^{+}} \frac{1}{t}\left(\int_{\Omega(t)} v(x) f(x) d x-\int_{\Omega(0)} v(x) f(x) d x\right),
$$


where

$$
\Omega(t)=\left\{x: v(x)+t h(x)-v_{c}>0\right\} .
$$

This suggests to use the speed method from shape optimization, see e.g. [13], to compute the shape derivative of $j$. We obtain the following result.

Proposition 4.1. The shape derivative of $j$ at $v$ in direction $h$ can be expressed as

$$
d j(v ; h)=\int_{\Gamma_{0}} \frac{h(x)}{|\nabla v(x) \cdot n|} f(x) d x,
$$

where $n$ denotes the outer unit normal to the set $\left\{x: v(x) \geq v_{c}\right\}$ and we assume that $\nabla v(x) \neq 0$ in a neighborhood of $\Gamma_{0}=\left\{x: v(x)=v_{c}\right\}$.

The evolution of the boundaries $\Gamma(t)=\left\{x: v(x)+t h(x)-v_{c}=0\right\}$ of $\Omega(t)$ can be seen to satisfy

$$
\nabla v(x(t)) \cdot \dot{x}(t)+h(x(t))+t \nabla h(x(t)) \cdot \dot{x}(t)=0 .
$$

For the speed method we assume that $t \rightarrow x(t)$ is a flow determined by a dynamical system of the form $\dot{x}(t)=\tilde{V}(x(t))$. In the particular case of a flow along the normal direction, which we follow here, we take $\tilde{V}(x)=-V(x) \frac{\nabla v(x)}{|\nabla v(x)|}$, for a scalar-valued function $V$ determined from (26). We find

$$
-V(x(t))|\nabla v(x(t))|+h(x(t))-t V(x(t)) \frac{\nabla v(x(t)) \cdot \nabla h(x(t))}{|\nabla v(x(t))|}=0
$$

or

$$
V(x(t))=\frac{h(x(t))}{|\nabla v(x(t))|+t \nabla(x(t)) \frac{\nabla v(x(t))}{|\nabla v(x(t))|}} .
$$

The shape derivative of $j(v)$ at $v$ in direction $h$ is therefore given by see [13], pg. 348,

$$
d j(v ; h)=\int_{\Gamma_{0}} \tilde{V}(x) \frac{-\nabla v(x)}{|\nabla v(x)|} f(x)=\int_{\Gamma_{0}} \frac{h(x)}{|\nabla v(x)|} f(x) d x,
$$

where we use that $v(x(t))=v(x)$ on $\Gamma_{0}$ for $t=0$. Since the tangential component of $v$ on $\Gamma_{0}$ is zero the shape derivative can equivalently be expressed in the form (25), as claimed above.

We illustrate this result with an example. We consider the one-dimensional domain $\Omega=(-1,1) \subset \mathbb{R}^{1}$, with $v_{c}=0$, and $h=1$, so that the boundary $\Gamma$ results in those points in $(0,1)$, where $g(v(x))=0$. The results for the analogue of the one-dimensional modification to (26) for different choices of $v(x)$ and $f(x)$, with $h=1$ are given in the third column of Table 4 . These results are compared to those obtained by numerical differentiation:

$$
I_{\epsilon}=\frac{1}{2 \epsilon}\left(\int_{\Omega} g(v(x)+\epsilon) \cdot f(x) d x-\int_{\Omega} g(v(x)-\epsilon) \cdot f d x\right) .
$$

in columns 4-6 of the same table. We can observe the expected convergence. 


\begin{tabular}{cc||c||c|c|l}
\multicolumn{1}{c||}{} & \multicolumn{1}{c||}{ shape derivative } & \multicolumn{3}{c}{ numerical differentiation } \\
$v$ & $f$ & formulation & $\epsilon=0.1$ & $\epsilon=0.01$ & $\epsilon=0.001$ \\
\hline $\cos (x \pi)$ & $x+1$ & $\frac{2}{\pi} \approx 0.63661$ & 0.63768 & 0.63663 & 0.63662 \\
$\cos (x \pi)$ & $x^{2}+x+3$ & $\frac{13}{2 \pi} \approx 2.06901$ & 2.08286 & 2.07006 & 2.06911 \\
$\cos \left(x^{2} \pi\right)$ & $x+1$ & $\frac{\sqrt{2}}{\pi} \approx 0.45015$ & 0.44394 & 0.44945 & 0.45008 \\
$\cos \left(x^{2} \pi\right)$ & $x^{2}+x+3$ & $\frac{7 \sqrt{2}}{2 \pi} \approx 1.57555$ & 1.56084 & 1.57379 & 1.57537
\end{tabular}

Table 1: Verification of the shape derivative formula (27). Different test cases of $v(x)$ and $f(x)$ are evaluated and compared to the numerical differentiation with different choices of $\epsilon$.

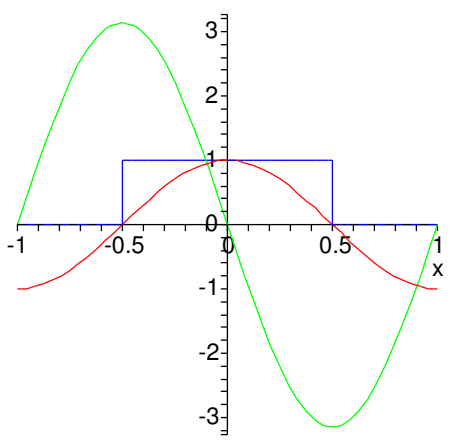

(a) $v_{1}=\cos (x \pi)$

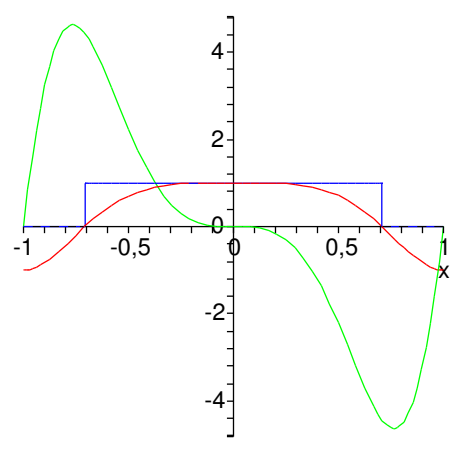

(b) $v_{2}=\cos ^{2}(x \pi)$

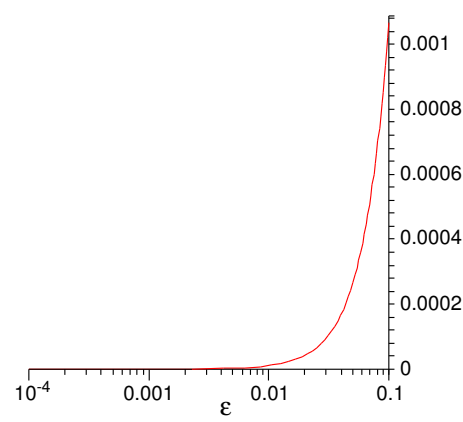

(c) $\left|d j-I_{\epsilon}\right|$ for $\epsilon \rightarrow 0$

Figure 1: (a), (b): illustration of the different choices of $v(x)$ (red), the heaviside function (blue) and the derivative $v^{\prime}(x)$ (green). (c): convergence of the finite difference solution to our formulation, for the test case $v(x)=\cos (x \pi)$ and $f(x)=x+1$

\section{Discretization}

\subsection{Semi-discretization in space}

The primal and adjoint problems are solved by the finite element method. Thus, the weak solution triple $(u, v, f, s)$ satisfies for a.e. $t \in(0, T)$ and for all $\varphi \in H^{1}(\Omega)$

$$
\begin{aligned}
0 & =\left\langle\nabla \cdot\left(\sigma_{i}+\sigma_{e}\right) \nabla u+\nabla \cdot \sigma_{i} \nabla v, \varphi\right\rangle \\
\left\langle\frac{\partial v}{\partial t}, \varphi\right\rangle & =\left\langle\nabla \cdot \sigma_{i} \nabla v+\nabla \cdot \sigma_{i} \nabla u-\left(I_{f i}+I_{s o}+I_{s i}\right)+I_{t r}, \varphi\right\rangle, \\
\left\langle\frac{\partial f}{\partial t}, \varphi\right\rangle & =\langle F(v, f), \varphi\rangle \\
\left\langle\frac{\partial s}{\partial t}, \varphi\right\rangle & =\langle S(v, s), \varphi\rangle
\end{aligned}
$$

together with initial and boundary conditions (10)-(13). Let $V_{h} \subset H^{1}(\Omega)$ be the finite dimensional subspace of piecewise linear basis functions with respect to the spatial grid. The approximate solutions $\mathbf{u}, \mathbf{v}, \mathbf{f}$ and $\mathbf{s}$ are expressed in the form $\mathbf{u}(t)=\sum_{i=1}^{N} u_{i}(t) \omega_{i}$, $\mathbf{v}(t)=\sum_{i=1}^{N} v_{i}(t) \omega_{i}, \mathbf{f}(t)=\sum_{i=1}^{N} f_{i}(t) \omega_{i}$ and $\mathbf{s}(t)=\sum_{i=1}^{N} s_{i}(t) \omega_{i}$, respectively, where $\left\{\omega_{i}\right\}_{i=1}^{N}$ denote the basis functions. The semi-discretization of primal equations in space 
results in the differential algebraic system of the form:

$$
\begin{aligned}
\mathbf{A}_{\mathbf{i e}} \mathbf{u}+\mathbf{A}_{\mathbf{i}} \mathbf{v} & =\mathbf{I}_{e}, \\
\mathbf{M} \frac{\partial \mathbf{v}}{\partial t} & =-\mathbf{A}_{\mathbf{i}} \mathbf{v}-\mathbf{A}_{\mathbf{i}} \mathbf{u}-\left(\mathbf{I}_{f i}+\mathbf{I}_{s i}+\mathbf{I}_{s o}\right)+\mathbf{I}_{t r}, \\
\frac{\partial \mathbf{f}}{\partial t} & =\mathbf{F}(\mathbf{v}, \mathbf{f}), \\
\frac{\partial \mathbf{s}}{\partial t} & =\mathbf{S}(\mathbf{v}, \mathbf{s}),
\end{aligned}
$$

together with initial conditions for $\mathbf{v}, \mathbf{f}$ and $\mathbf{s}$, where $\mathbf{A}_{\mathbf{i e}}=\left\{\left\langle\left(\sigma_{i}+\sigma_{e}\right) \nabla \omega_{i}, \nabla \omega_{j}\right\rangle\right\}_{i, j=1}^{N}$ and $\mathbf{A}_{\mathbf{i}}=\left\{\left\langle\sigma_{i} \nabla \omega_{i}, \nabla \omega_{j}\right\rangle\right\}_{i, j=1}^{N}$ are the stiffness matrices, $\mathbf{M}=\left\{\left\langle\omega_{i}, \omega_{j}\right\rangle\right\}_{i, j=1}^{N}$ is the mass matrix, and the vectors $\mathbf{I}_{e}, \mathbf{I}_{i t r}$ are defined by $\mathbf{I}_{e}=\left\{\left\langle\left(\chi_{\partial \Omega_{1}} I_{e}-\chi_{\partial \Omega_{2}} I_{e}\right), \omega_{j}\right\rangle\right\}_{j=1}^{N_{\partial \Omega}}$ and $\mathbf{I}_{t r}=\left\{\left\langle I_{t r}, \omega_{j}\right\rangle\right\}_{j=1}^{N}$, respectively. The expressions for $\mathbf{I}_{f i}(\mathbf{v}, \mathbf{f}), \mathbf{I}_{s i}(\mathbf{v}, \mathbf{s})$ and $\mathbf{I}_{s o}(\mathbf{v})$ are defined by

$$
\begin{gathered}
\mathbf{I}_{f i}(\mathbf{v}, \mathbf{f})=\left(\mathbf{I}_{f i}\right)\left(\sum_{i=0}^{N} v_{i} \omega_{i}, \sum_{i=0}^{N} f_{i} \omega_{i}\right), \\
\mathbf{I}_{s i}(\mathbf{v}, \mathbf{s})=\left(\mathbf{I}_{s i}\right)\left(\sum_{i=0}^{N} v_{i} \omega_{i}, \sum_{i=0}^{N} s_{i} \omega_{i}\right), \text { and } \\
\mathbf{I}_{s o}(\mathbf{v})=\left(\mathbf{I}_{s o}\right)\left(\sum_{i=0}^{N} v_{i} \omega_{i}\right)
\end{gathered}
$$

respectively.

Remark 5.1. Specifically, $\mathbf{u}_{0}$ has zero mean throughout the optimization. Moreover, the iterative linear solver to obtain the current solution for (34) is initialized by the solution of the previous time-level. In this way, the compatibility condition for the singular algebraic system Eq. (34) is satisfied at each iteration level, i.e.

$$
\mathbf{c}^{T}\left(-\mathbf{A}_{\mathbf{i}} \mathbf{v}-\mathbf{I}_{e}\right)=-\left(\mathbf{c}^{T} \mathbf{A}_{\mathbf{i}}\right) \mathbf{v}-\mathbf{c}^{T} \mathbf{I}_{e}=0,
$$

where $\mathbf{c}=(1, \ldots, 1)^{T}$ is a constant vector. Analogously, the zero mean property is satisfied at every time-level of the iterative procedure for solving the dual elliptic equation.

\subsection{Time discretization}

The semi-discretization of the Eq. (35) and of the adjoint equation (20) after space discretization can be expressed in the following general form,

$$
\mathbf{M} \frac{\partial \mathbf{x}}{\partial t}=\mathbf{F}(\mathbf{x}), \quad \mathbf{x}\left(t^{0}\right)=\mathbf{x}^{\mathbf{0}} .
$$

To solve (38), we introduce discrete steps:

$$
0=t^{0}, t^{1}, \ldots, t^{n}=T,
$$

which are not necessarily equidistant. For the time discretization we employ the linearly implicit Rosenbrock method to solve the Eq. (38). This belongs to a large class of methods which avoid the nonlinear system and replace it by a sequence of linear ones. Here we applied a third order Rosenbrock method called ROS3PL [19]. For details we refer to [9, Section 3.2]. 


\subsection{Solution procedure}

We describe the solution procedure to solve the primal system by decoupling the system as follows.

Step 1: Use the available solution $\mathbf{v}^{i}$ at time $t^{i}$, solve the ODE equations (36), (37) for $\mathbf{f}^{i+1}$ and $\mathbf{s}^{i+1}$ at time $t^{i+1}$ by using the explicit Euler method.

Step 2: Use the solution $\mathbf{v}^{i}$ at time $t^{i}$, solve the discretized elliptic system (34) for $\mathbf{u}^{i+1}$ at time $t^{i+1}$ by using a stabilized saddle point approach.

Step 3: Finally, by utilizing the computed solutions $\mathbf{u}^{i+1}, \mathbf{f}^{i+1}$ and $\mathbf{s}^{i+1}$, solve the discretized parabolic equation (35) for $\mathbf{v}^{i+1}$ at time $t^{i+1}$ by applying the linearly implicit RungeKutta method.

To solve the gating equations, we use the explicit Euler method which is cheaper in terms of CPU time compared to implicit methods. The solutions of the singular linear systems which arise after the full discretization of Eqs (1) and (19) are defined up to an additive constant. We mentioned earlier that we impose a zero mean condition to fix this constant. For the numerical realization of this condition we adopted a stabilized saddle point formulation from the work of Bochev and Lehoucq [7]. The discussion and implementation details of this technique for the current problem we refer to $[9,10]$. To solve the linear system we employed a BiCGSTAB [35] method with AMG preconditioner [6], which is developed using a greedy heuristic algorithm for the aggregation based on a strength of connection criterion. After the full discretization of (35) we obtain a system of linear algebraic equations. To solve this linear system we employed a BiCGSTAB method with Jacobi preconditioning.

The complete optimality system is solved by the non-linear conjugate gradient (NCG) [23] combined with the Hager-Zhang variant update. It is well known that gradient based algorithms exhibit slow convergence. In spite of that, due to their simple implementation they are the method of choice to numerically verify that the proposed optimal control approach is feasible. A line search procedure based on the Armijo rule with backtracking is used to determine the updates during the optimization iterations.

\subsection{Parallel implementation}

In the context of optimal control of the bidomain equations the necessity of repeatedly solving the coupled forward-backwards systems of primal and adjoint equations for possibly very different inputs representing the controls, calls for using a parallel implementation, which in our case is combined with a nonlinear conjugate gradient algorithm for solving the optimal control problem. Recent research work on parallel tools and high performance computing for the bidomain equations is devoted simulation of fine scale spatio-temporal simulations with sufficient efficiency, see e.g. [25, 36, 24, 22, 31].

In our simulations we used the software package DUNE [2], especially the dune-pdelab [4] discretization module, which is a $\mathrm{C}++$ template based programming environment for solving a general class of PDE's. The internal parallel Cartesian (called Yasp) grid in DUNE is used for parallel grid constructions. For the domain decomposition technique the original domain is partitioned into sub domains and each sub domain is assigned to a single processor. In our computations, we used a non overlapping domain decomposition to solve the primal and adjoint equations.

\subsection{Integrals along iso surfaces}

As described in Section 4 it is necessary to evaluate integrals along the iso surface of the control variable. This is achieved via a polygonal reconstruction of the iso surface and is 


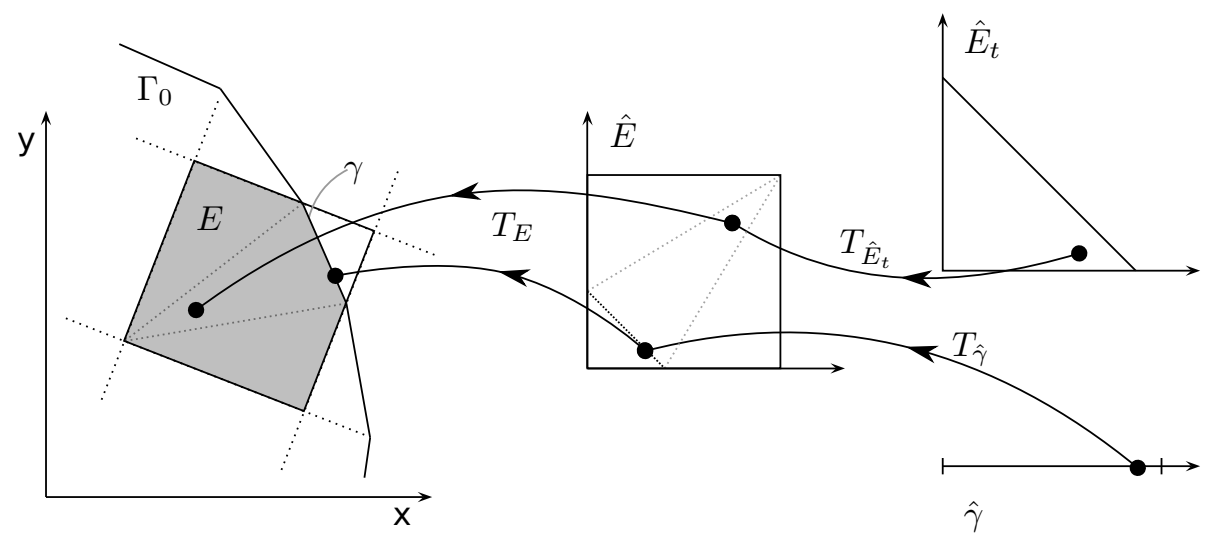

Figure 2: Mesh cell $E$, its reference element $\hat{E}$ and the local triangulation used for the interface reconstruction, with the reference line $\hat{\gamma}$, or the reference triangle $\hat{E}_{t}$. Integration along the edge $\gamma \subset \Gamma_{0}$ is constructed by concatenation of the transformations $T_{E}$ from the reference element to global coordinates and $T_{\hat{\gamma}}$ from the reference line to $E$.

exact for piecewise linear functions. For the integration we use the dune-mc library [16], which provides a local reconstruction of the interface. The library implements an extended marching cubes 33 algorithm [11], which constructs a local triangulation of the interface, the two adjacent domains and the local connectivity graph. The reconstruction of the local interface yields a set of geometric entities $\gamma$, for which standard Gauss quadrature rules can be employed.

On each cell of the global mesh we consider vertex values $v_{i}=v\left(x_{i}\right)$. We generate a lookup-key by assigning $\left(v_{i} \geq v_{c}\right)$ to the i-th bit of an integer

$$
k=\sum_{i}\left(v_{i} \geq v_{c}\right) \cdot 2^{i}
$$

Look up tables store for each key a unique reconstruction or a particular rule to disambiguate this situation, which then points to the disambiguated reconstruction. From the lookup the library computes a set of lines (in 2D) or triangles (in 3D) and the position of their vertices $\xi_{i}$. The library guarantees different topological consistencies, which are necessary for numerical algorithms

1. The connectivity pattern of the cell vertices are preserved within each sub-entity of the mesh cell. In particular this means that vertices connected along an edge, face or volume, are still connected via the same sub-entity after the construction.

2. The exact interface $\Gamma_{0}=\left\{x: v(x)=v_{c}\right\}$ partitions each grid cell into patches belonging to two sub domains $\left.\Omega\right|_{g=0}$ or $\left.\Omega\right|_{g=1}$. In particular this means that the number of patches and their domain association is the same for the polygonal reconstruction.

3. The vertices of the reconstructed interface lie on the exact interface, i.e. for each vertex $\xi_{i}$ the property $v_{h}\left(\xi_{i}\right) \equiv v_{c}$ holds.

Using a co-dimension 1 Gauss quadrature rule $(q, \omega)$ (e.g. a 1D rule in 2D), with quadrature points $q$ and weights $\omega$, the integral along the interface is evaluated as

$$
\int_{\Gamma_{0, h}} \frac{1}{|\nabla v(x)|} f(x) d x=\sum_{\gamma} \sum_{(q, \omega)} \omega \sqrt{\operatorname{det}\left(J_{E} J_{\hat{\gamma}} J_{\hat{\gamma}}^{T} J_{E}^{T}\right)} \frac{1}{\left|J_{E}^{-1} \sum_{j} v_{j} \nabla \phi_{j}(q)\right|} f(q),
$$

where $J_{E}$ denotes the Jacobin of the transformation $T_{E}$ and $J_{\hat{\gamma}}$ that of $T_{\hat{\gamma}}$. The integration volume is given as $\sqrt{\operatorname{det}\left(J_{E} J_{\hat{\gamma}} J_{\hat{\gamma}}^{T} J_{E}^{T}\right)}$, based on the concatenation of the transformations 
$T_{\hat{\gamma}}$ and $T_{E}$. The gradient of $v(x)$ is evaluated in local coordinates of the reference element $E$ and transformed into global coordinated by multiplying with the inverse of the Jacobin. The different transformations are depicted in Figure 2.

Assembling the local stiffness matrix requires the evaluation of integrals restricted to a mesh cell. In addition to the standard term for the volume integrals we also evaluate the integral along the interface segment $\gamma$, this is approximated in the following way:

$$
\begin{aligned}
\int_{\gamma} \frac{\left(I_{f i}\right)_{v}+\left(I_{s o}\right)_{v}}{|\nabla v|} & \phi_{i} \phi_{j} d x= \\
& \sum_{(q, \omega)} \omega \sqrt{\operatorname{det}\left(J_{E} J_{\hat{\gamma}} J_{\hat{\gamma}}^{T} J_{E}^{T}\right)} \frac{\left(I_{f i}\right)_{v}+\left(I_{s o}\right)_{v}}{\left|J_{E}^{-1} \sum_{k} v_{k} \nabla \phi_{j}(q)\right|} \phi_{i}(q) \phi_{j}(q) .
\end{aligned}
$$

This procedure takes place on each individual element of the global mesh during the assembly of the matrices. In parallel computations, the assembly of such terms is carried out without requiring any further communication, as the construction is purely local.

\section{Numerical Results}

The computational domain is $\Omega=(0,5) \times(0,5) \subset \mathbb{R}^{2}$ of size $5 \times 5 \mathrm{~cm}^{2}$ and a $256 \times 256$ uniform quadrilateral spatial grid is used which consists of 65,536 elements and 66,049 nodes. Thus the computation involved 264,196 dofs for one PDE solve. In our computations, the stopping criteria for the optimization algorithm is based on the following conditions.

$$
\left\|\nabla J\left(\mathbf{I}_{e}^{k}\right)\right\|_{L^{2}} \leq 10^{-5} \cdot\left|J\left(\mathbf{I}_{e}^{k}\right)\right| \text { or }\left|J\left(\mathbf{I}_{e}^{k}\right)-J\left(\mathbf{I}_{e}^{k-1}\right)\right| \leq 10^{-4} .
$$

If these conditions are not satisfied within a prescribed number of 200 iterations, the algorithm is terminated.

First numerical results based on boundary control of the bidomain equations are presented where the control acts on the left and right boundary of the computational domain, as shown in Figure 3.

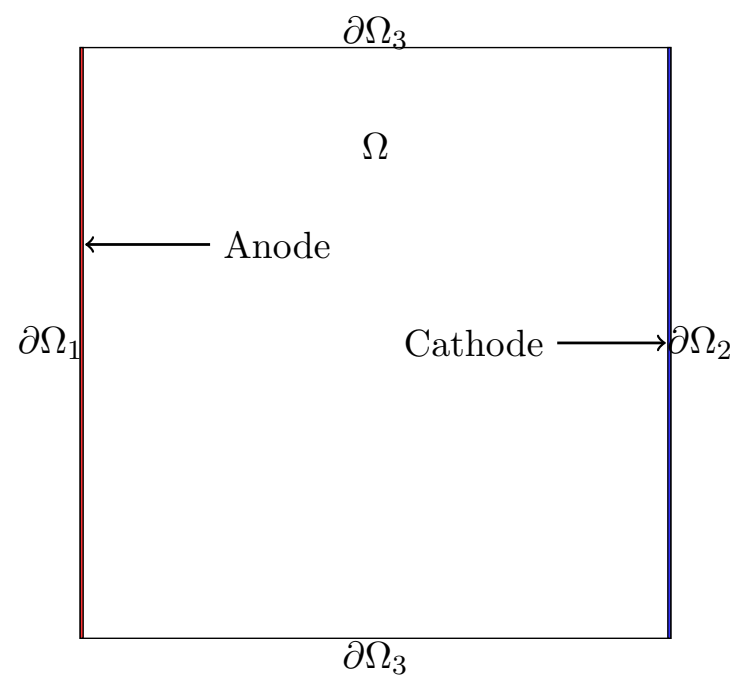

Figure 3: The computational domain setup with stimulation boundaries.

In pathological states, structural discontinuous in the cardiac tissue play a crucial role and can interfere with the spread of cardiac activation and contribute to initiation of spiral waves and wave break $[12,14]$. In our study, the structural heterogeneity is generated 
based on the histological image, see left panel of Figure 4. First we convert the histological image to gray scale image, right panel of Figure 4. Then 0's and 1's are generated based on this gray scale image. The generated 0's and 1's information is projected onto the computational mesh. Subsequently, the conductivity tensor values are reduced by $10 \%$ in all finite element cells marked by 1's. The resulting conduction velocities are 0.54 and 0.39 in the longitudinal and transverse direction, respectively. The action potential duration was $98 \mathrm{~ms}$ using the given parameters in Table 2.

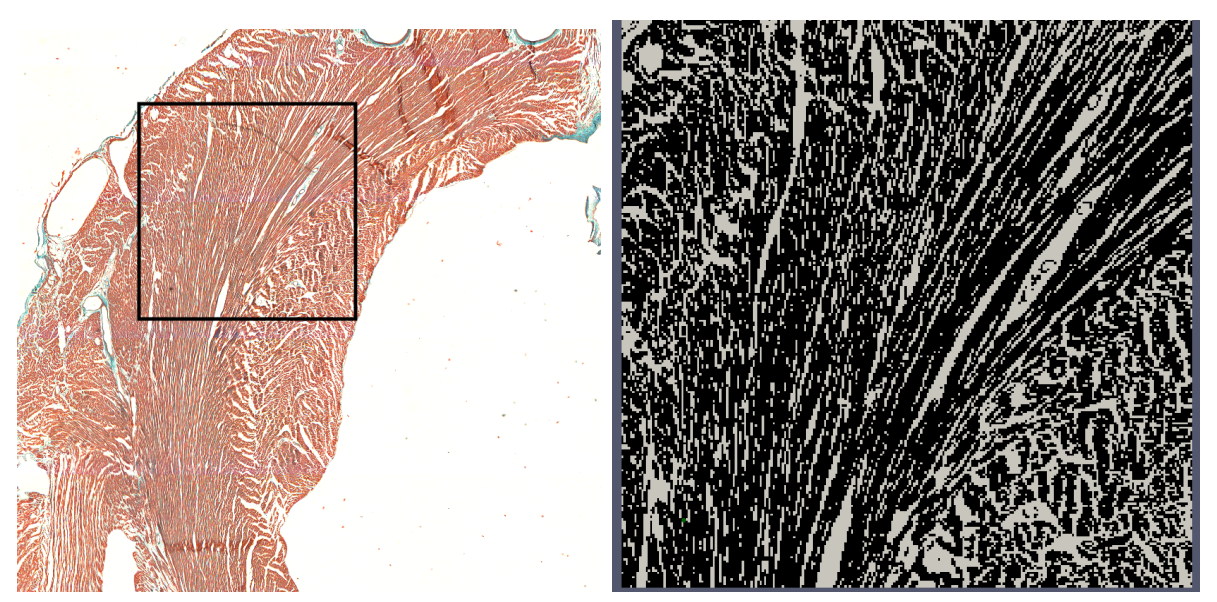

Figure 4: Histological image of the cardiac tissue in the left panel [8]. The selected region in the histological image converted to a gray scale image in the right panel.

Concerning initialization, a standard $S 1-S 2$ stimulation protocol was applied to induce a reentrant activation pattern. An initial $S 1$ stimulus of $I_{t r}=100 \mu \mathrm{A} / \mathrm{cm}^{3}$ and $5 \mathrm{msec}$ duration was applied at time $t=0 \mathrm{msec}$ along the bottom edge of the tissue sheet. At time $t=183 \mathrm{msec}$, when the critical recovery isoline crossed the center of the sheet, a second $S 2$ stimulus of $I_{t r}=100 \mu \mathrm{A} / \mathrm{cm}^{3}$ and $5 \mathrm{msec}$ duration was applied in a small region of $0.3 \mathrm{~cm}$ radius at the center of the domain. This $S 2$ stimulus generated two phase singularities at the intersections between critical recovery isoline and the boundary of the $S 2$ stimulus region, leading to a so-called Figure of Eight reentrant pattern. The solution at $t=435 \mathrm{msec}$ was then chosen as the initial state for simulating the delivery of electrical shocks and the post-shock evolution following at the end of the shock.

The three temporal horizons are illustrated in Figure 5. The solution for the transmembrane voltage $v$ in absence of any control is shown in Figure 10 at different time instances to demonstrate that the reentry is sustained. While during the shock, which constitutes the time period within which optimization takes place, the size of the time step was kept constant at $\Delta t=0.04 \mathrm{msec}$, adaptive time stepping of the ROS3PL method was used in the pre- and post-shock phases.

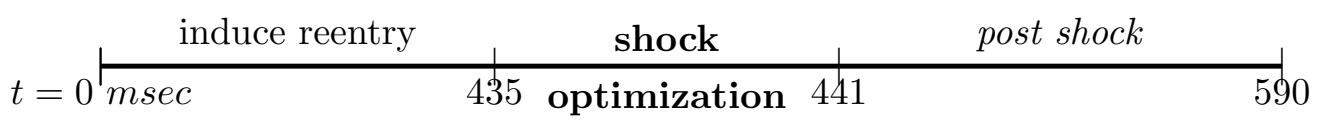

Figure 5: The time horizons.

\subsection{Termination of reentrant waves}

The desired trajectory of the transmembrane potential $\left(v_{d}\right)$ in $(15)$ plays a crucial role for successful defibrillation. In our computations, this is done by solving once the primal problem using a prescribed time course of a stimulation current, $I_{e}(t)=0.2 \mathrm{~mA} / \mathrm{cm}^{3}$. The 
optimal control procedure then determines a stimulus which still leads to defibrillation but with less energy.
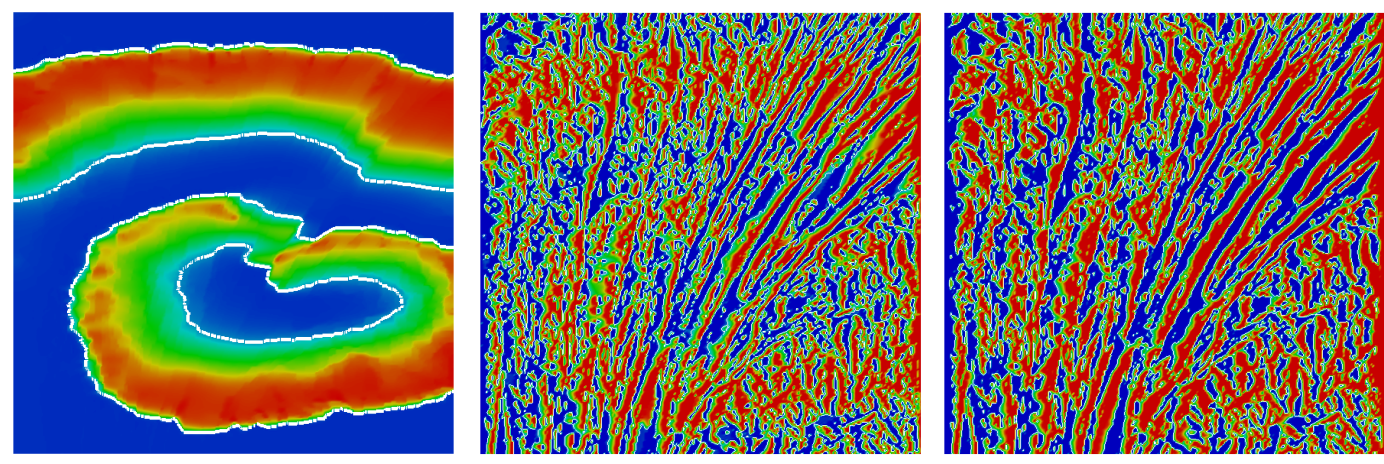

Figure 6: Interface tracking of the controlled transmembrane voltage solution during the shock period at time $t=435 \mathrm{msec}$ at left, $t=437 \mathrm{msec}$ at middle and $t=441 \mathrm{msec}$ at right respectively. White lines represents the interface of function $g(v), \alpha_{1}=0.05$ and $\alpha_{2}=10$.

In Figure 6 we depict the optimally controlled transmembrane voltage during the shock period. We can see clearly a large number of virtual electrodes appear shortly after delivering the suitable stimulus strength. Primarily, they appear along the fiber direction on the computational domain, see in middle and right of Figure 6 . The reconstructed iso-surface at the critical value is depicted with white lines on the cardiac tissue. Here we observed that the marching cube algorithm implementation library [16] detected all those large number of electrodes.

The gradient value of the cost functional and associated values of the cost for $\alpha_{1}=0.05$ and different values for $\alpha_{2}$ are given on the left of Figure 7, as a function of the NCG iterations. As expected, the values of the cost do not tend to zero. First, there is a negligible control cost and, secondly, we certainly do not expect $v$ to follow $v_{d}$ exactly. Optimal controls for different values of $\alpha_{2}$ are depicted on the right of Figure 7. Successful defibrillation is achieved for $\alpha_{2} \leq 40$, with smaller stimulus strength than that used for generating $v_{d}$ which is $0.2 \mathrm{~mA} / \mathrm{cm}^{3}$. The subsequent results are obtained with $\alpha_{2}=10$.
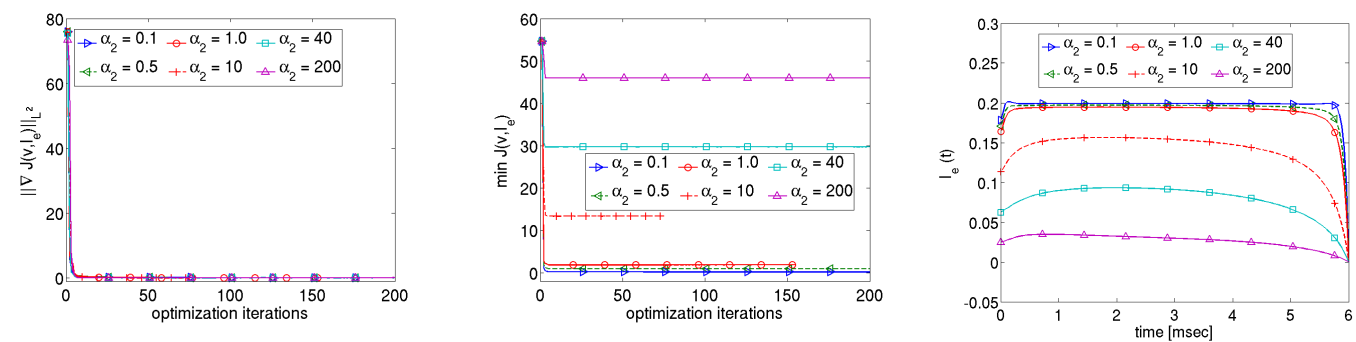

Figure 7: The minimum value of the cost functional and the optimal controls on the left and right respectively with $\alpha_{1}=0.05$ and varying $\alpha_{2}=10$.

The optimally controlled state variables are presented in Figure 8 by plotting the solution at the horizontal line extending from $(0,3)$ to $(5,3)$, for different time instances. The first panel depicts the optimal extracellular potential which is small towards the end of the control interval. The corresponding transmembrane potential value is plotted in second panel of Figure 8, where one can see depolarization and hyperpolarization effects. We observe that virtual electrodes appear randomly and the polarization value increases with time. At the end of the shock period depolarization and hyperpolarization sustain for a while before they also tend to zero. The virtual electrodes which have a big impact on 
the fast and slow gating variables, are depicted in the third and fourth panels of Figure 8 respectively. The difference between the maximum and minimum value of the fast gating variable is large compared to the effect in the slow gating variable.
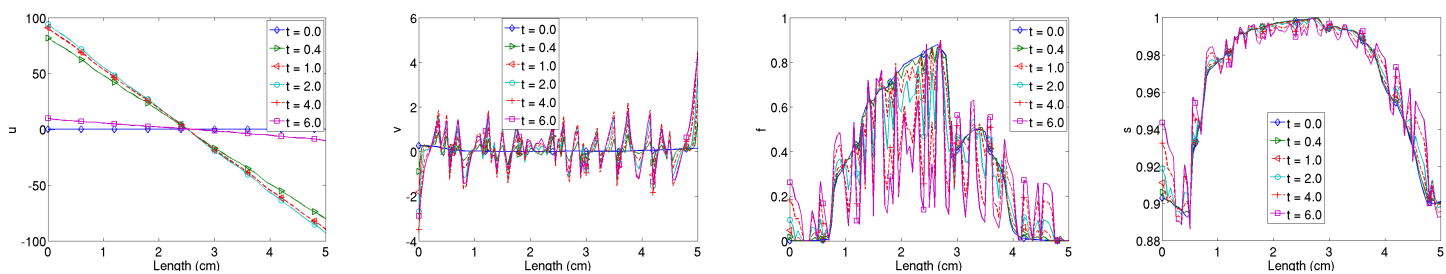

Figure 8: Optimal states versus time.

The post shock simulation results of the state variables are shown in Figure 9 as a function of $x_{1}$ at $y_{1}=3.0$. The extracellular potential decays rapidly due to absence of control input for the source function. The transmembrane voltage simulation takes a while to disappear due to virtual electrodes. Since most of the computational domain is excited at the end of shock period further excitation of the wave propagation is blocked and $v$ goes to rest at the end of the simulation. Similarly, the gating variables also take a while to smoothing out before going to the resting state, see third and forth panels of Figure 9 .
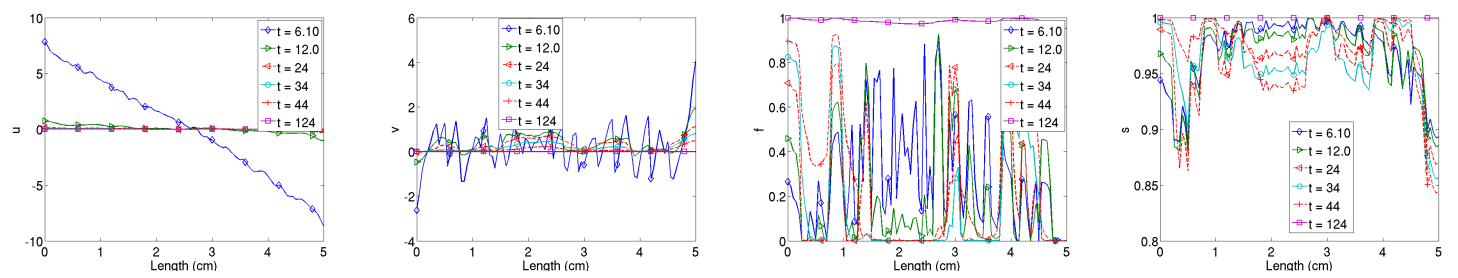

Figure 9: Optimal states during post shock period.

We can now compare the spatial representation of the uncontrolled solution $u$ in Figure 10 to the controlled solution in the post shock phase in Figure 11. Clearly, the wave propagation almost disappears at 539 msec of simulation time. It disappears completely after 547 msec.
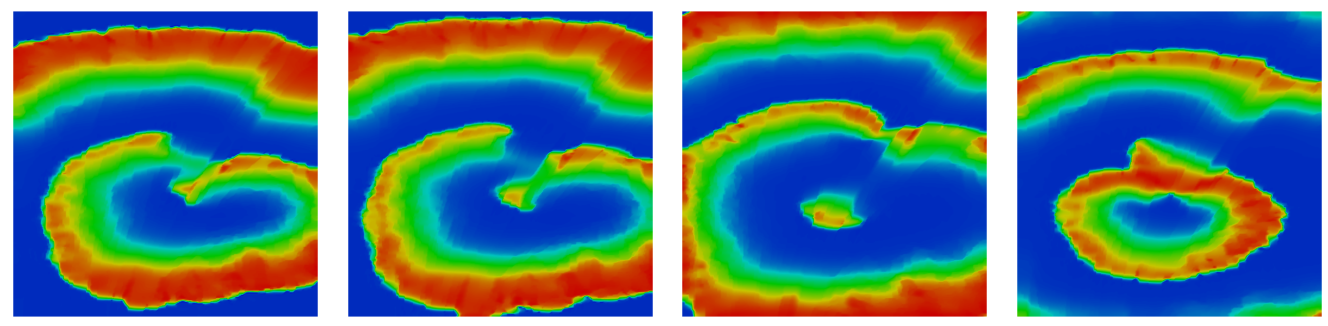

Figure 10: Uncontrolled solution of transmembrane voltage at times $t=443,453,481$ and 539 msec respectively.

\subsection{Numerical test with mesh dependency}

We discuss the robustness of the optimization algorithm with respect to spatial grid refinement. Uniform Cartesian grids of size $128 \times 128,256 \times 256,512 \times 512$ as well as $1024 \times 1024$ were considered. First, the reentrant solution which is used as the initial solution for optimization is generated on the $128 \times 128$ grid and, to eliminate possible later effects on robustness the solution on finer grids is obtained by linear interpolation. The conductivity tensors are generated directly form the histological mesh and the desired 

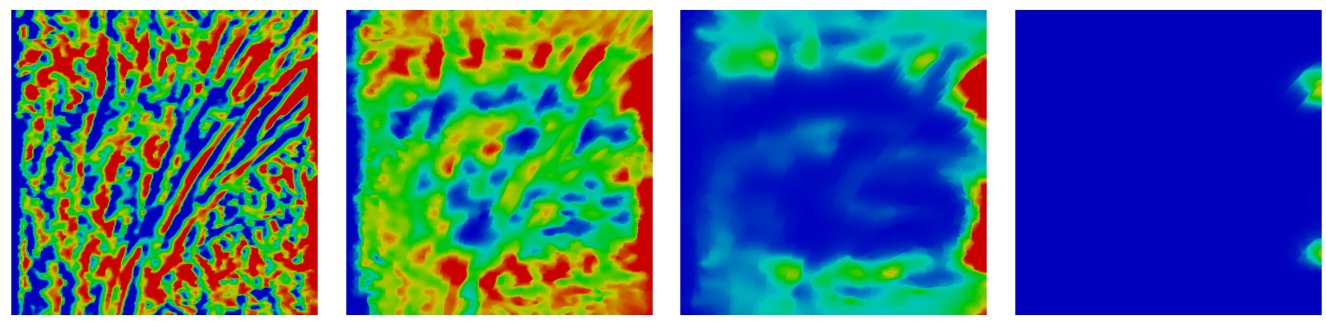

Figure 11: Controlled solution of transmembrane voltage during the post shock simulations at times $t=443,453,481$ and 539 msec respectively.

trajectory of the transmembrane voltage is constructed, each on different grid resolutions. The optimal controls for these different mesh sizes are depicted in Figure 12. Apparently, the optimal controls converge as the grid is refined.

The total value of the current $\int_{0}^{T} I_{e}(t) d t$ is $2.5262(\mathrm{~mA} \mathrm{~ms}) / \mathrm{cm}^{3}, 2.8352(\mathrm{~mA} \mathrm{~ms}) / \mathrm{cm}^{3}$, $2.8794(\mathrm{~mA} \mathrm{~ms}) / \mathrm{cm}^{3}$ and $2.9020(\mathrm{~mA} \mathrm{~ms}) / \mathrm{cm}^{3}$ for these four mesh sizes respectively, which is clearly less than that used to generate $v_{d}$. Still, the desired defibrillation is achieved in all cases.

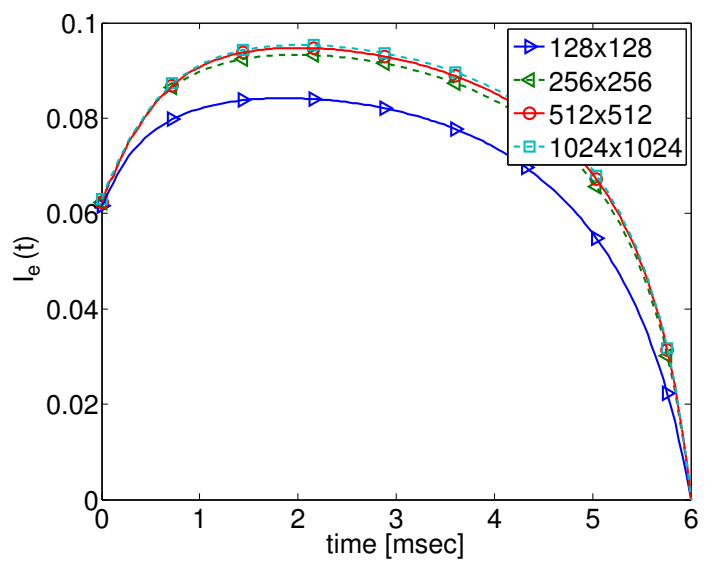

Figure 12: Optimal control for different mesh sizes.

\subsection{Parallel efficiency}

The computational cost in an optimal control problem mainly depends on the PDE solves for the primal and dual systems. Here we demonstrate the strong scalability of of the PDE solve as well as the complete optimization problem. To assess the strong scaling properties of the solvers used in this study benchmark simulations were performed using a sufficiently fine resolution of a square $2 \mathrm{D}$ domain of size $5 \times 5 \mathrm{~cm}$ which consisted of 262,144 elements and 263,169 nodes $(512 \times 512$ grid). The optimization computation was restricted to 10 optimization iterations for better comparison of the computational time over the different number of processors.

The parallel efficiency was measured by

$$
e=\frac{1}{N} \frac{T_{1}}{T_{N}}
$$

where $T_{N}$ is the total CPU time of a reference simulation on a single core and $N$ and $T_{N}$ are the number of cores and the total CPU time in the scalability experiments.

The parallel efficiency of this benchmark was demonstrated for different components of the solver as well as for the complete optimization. Here we present the parallel efficiency in 
the left panel of Figure 13 for the finite element method assembly of the left hand side of the matrix in the Rosenbrock time stepping method which needs to be computed in each time step, and for the solution of elliptic, parabolic and ode problems. For these components, an average time on total processors was measured to compute the parallel efficiency. We can observe that solving the ODE scales very well up to 64 processors, since almost no communication for this task is required. Only the solution of the elliptic solve scales poorly on 64 processors. This can be attributed to the unfavorable surface-to-volume ratio of local domains. With increasing $N$, the relation between local compute work performed on inner nodes of the domain and the data communication which are proportional to the size of shared domain interfaces, deteriorates, thus impeding any further efficient scaling. The domain sizes with 32 and 64 cores in terms of nodes were 8385 and 4225 only. The parabolic solver scales better than the elliptic solver. The CPU times on a single core for assembling the matrix is 198.29 seconds, the solution of the ODE takes 0.55 seconds, the parabolic solve needs 364.51 seconds, the elliptic solve uses 1302.50 seconds, and the complete primal solve requires 2126.8 seconds. The dual problem requires 1876.87 seconds, the complete optimization algorithm needs 47741.5 seconds and the post shock simulation takes 12813.2 seconds. We observed that the elliptic solver requires $61.2 \%$ of the overall computational time in the primal solve.

The BiCGSTAB with Jacobi preconditioner takes an average of 13 iterations at each time step of the primal solve for the solution of parabolic problem for 1 up to 64 cores. Also the parabolic part in the dual solve took an average of 13 iterations. For the solution of the elliptic part in the primal problem, the BiCGSTAB with AMG preconditioning required 13 iterations on 1 core and on 64 cores it needed 25 iterations. For the elliptic equation in the dual problem, an average of 12 iterations on 1 core and an average of 22 iterations on 64 cores were required.

Furthermore, the efficiency for one primal and one dual solve, for the optimization algorithm as well as for the post simulation are depicted in the right panel of the Figure 13. Here we can observe that these components scale well up to 32 cores and poorly on 64 cores.
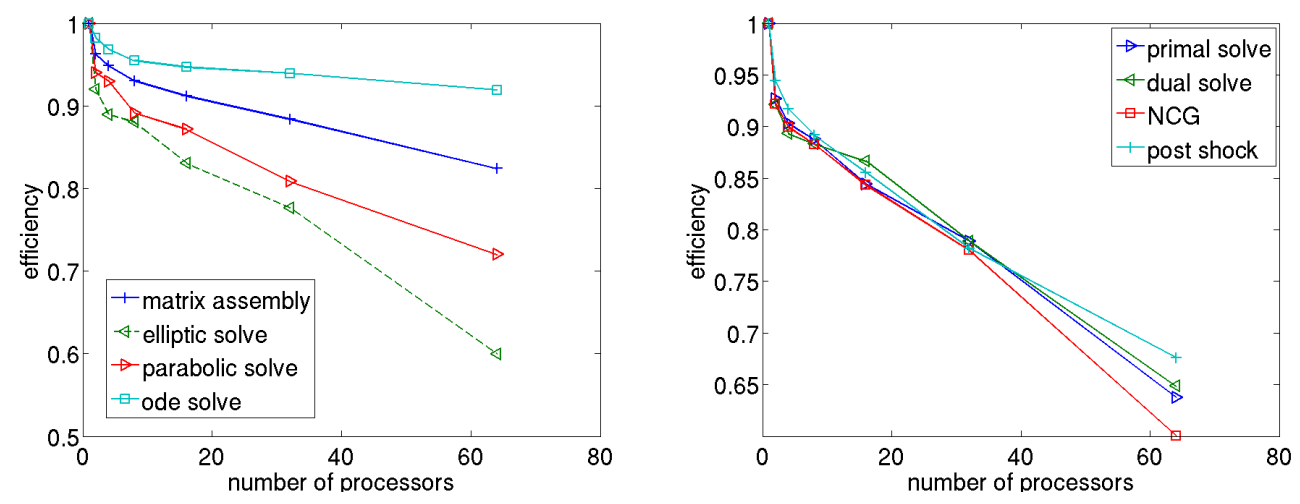

Figure 13: Parallel efficiency for the PDE solve and optimization computation.

\section{Conclusion}

We presented an optimal control technique for achieving successful defibrillation of reentrant activation patterns in a $2 \mathrm{D}$ sheet of cardiac tissue by taking into account their dynamical systems behavior for proper choice of a cost functional. The conductivity tensor distribution was based on the histological images. The Fenton-Karma model presents a particular challenge due to a Heaviside side function which appears in the source term. It describes a switching behavior of the gating variable depending on the transmembrane 
voltage. The main steps to overcome the resulting difficulties consisted in using shape calculus techniques to derive the sensitivity equations and an extended marching cube algorithm [16] for efficient numerical treatment of the interfaces, including iso-isosurface integrations during the finite element assembly of matrices in the computational domain. We also discussed parallel efficiency for the complete optimization as well as individual components. These techniques are applicable for a wider class of phenomenological models describing the ionic behavior on electro-physiological heart models, where switching structure play an essential role.

\section{Acknowledgment}

The authors gratefully acknowledge the Austrian Science Foundation (FWF) for financial support under SFB 032, "Mathematical Optimization and Applications in Biomedical Sciences" and the Austrian Academy of Sciences (Ö̈W).

\section{A Appendix}

The simulation parameters which are used in our computations are given in the following table.

\begin{tabular}{|rr|cr|cr|cr|}
\hline$\sigma_{e l}$ & $3 e^{-3}$ & $v_{c}$ & 0.13 & $\tau_{f 1}^{-}$ & 19.6 & $\tau_{s}^{-}$ & 11.0 \\
$\sigma_{e t}$ & $1.9 e^{-3}$ & $v_{c}^{s i}$ & 0.85 & $\tau_{f 2}^{-}$ & 1000.0 & $\tau_{s}^{+}$ & 667.0 \\
$\sigma_{i l}$ & $3 e^{-3}$ & $\kappa$ & 10.0 & $\tau_{f}^{+}$ & 3.33 & $\tau_{0}$ & 8.3 \\
$\sigma_{i t}$ & $3.6 e^{-4}$ & $\tau_{f i}$ & 0.36 & $\tau_{s i}$ & 45.0 & $\tau_{r}$ & 50.0 \\
\hline
\end{tabular}

Table 2: Simulation parameters

\section{References}

[1] C. Anderson, N. Trayanova, and K. Skouibine. Termination of spiral waves with biphasic shocks: role of virtual electrode polarization. Journal of Cardiovascular Electrophysiology, 11(12):1386-1396, 2000.

[2] P. Bastian, M. Blatt, A. Dedner, C. Engwer, R. Klöfkorn, R. Kornhuber, M. Ohlberger, and O. Sander. A generic grid interface for parallel and adaptive scientific computing. Part II: implementation and tests in DUNE. Computing, 82(2):121138 , July 2008.

[3] P. Bastian and C. Engwer. An unfitted finite element method using discontinuous Galerkin. International Journal for Numerical Methods in Engineering, 79(12):15571576, 2009.

[4] P. Bastian, F. Heimann, and S. Marnach. Generic implementation of finite element methods in the distributed and unified numerics environment (DUNE). Kybernetika, 46(2):294-315, 2010.

[5] G. W. Beeler and H. Reuter. Reconstruction of the action potential of ventricular myocardial fibres. The Journal of Physiology, 268(1):177-210, June 1977.

[6] M. Blatt. A parallel algebraic multigrid method for elliptic problems with highly discontinuous coefficients. PhD thesis, Ruprechts-Karls-Universität Heidelberg, 2010.

[7] P. Bochev and R. B. Lehoucq. On the finite element solution of the pure neumann problem. SIAM Rev., 47:50-66, January 2005. 
[8] F. Campos, T. Wiener, A. Prassl, R. Weber dos Santos, D. Sanchez-Quintana, H. Ahammer, G. Plank, and E. Hofer. Electro-anatomical characterization of atrial microfibrosis in a histologically detailed computer model. Biomedical Engineering, IEEE Transactions on, $\mathrm{PP}(99): 1-1,2013$.

[9] N. Chamakuri, K. Karl, and P. Gernot. Optimal control approach to termination of re-entry waves in cardiac electrophysiology. Journal of Mathematical Biology, pages 1-30, 2013. 10.1007/s00285-012-0557-2.

[10] N. Chamakuri, K. Kunisch, and G. Plank. On boundary stimulation and optimal boundary control of the bidomain equations. Mathematical Biosciences, 245(2):206 $215,2013$.

[11] E. V. Chernyaev. Marching cubes 33: Construction of topologically correct isosurfaces. Technical Report 17, CERN, 1995.

[12] J. M. T. de Bakker and H. M. V. van Rijen. Continuous and discontinuous propagation in heart muscle. J Cardiovasc Electrophysiol, 17(5):567-573, May 2006.

[13] M. C. Delfour and J.-P. Zolésio. Shapes and geometries: analysis, differential calculus, and optimization. Society for Industrial and Applied Mathematics, Philadelphia, PA, USA, 2001.

[14] Z. J. Engelman, M. L. Trew, and B. H. Smaill. Structural heterogeneity alone is a sufficient substrate for dynamic instability and altered restitution. Circulation: Arrhythmia and Electrophysiology, 3(2):195-203, 2010.

[15] B. Engquist, A.-K. Tornberg, and R. Tsai. Discretization of dirac delta functions in level set methods. Journal of Computational Physics, 207(1):28-51, 2005.

[16] C. Engwer and A. Nüßing. Geometric integration over irregular domains with topologic guarantees. in preparation.

[17] F. Fenton and A. Karma. Vortex dynamics in three-dimensional continuous myocardium with fiber rotation: Filament instability and fibrillation. Chaos: An Interdisciplinary Journal of Nonlinear Science, 8(1):20-47, Mar. 1998.

[18] C. S. Henriquez. Simulating the electrical behavior of cardiac tissue using the bidomain model. Crit. Rev. Biomed. Eng., 21:1 77, 1993.

[19] J. Lang and D. Teleaga. Towards a fully space-time adaptive FEM for magnetoquasistatics. IEEE Transactions on Magnetics, 44(6):1238-1241, 2008.

[20] C. Luo and Y. Rudy. A model of the ventricular cardiac action potential: Depolarization, repolarization, and their interaction. Circ. Res., 68:1501-1526, 1991.

[21] C. Mitchell and D. Schaeffer. A two-current model for the dynamics of cardiac membrane. Bulletin of Mathematical Biology, 65(5):767-793, 2003.

[22] S. Niederer, L. Mitchell, N. Smith, and G. Plank. Simulating human cardiac electrophysiology on clinical time-scales. Front Physiol, 2:14, 2011.

[23] J. Nocedal and S. J. Wright. Numerical Optimization. Springer Verlag, New York, second edition edition, 2006.

[24] L. F. Pavarino and S. Scacchi. Multilevel additive schwarz preconditioners for the bidomain reaction-diffusion system. SIAM J. Sci. Comput., 31:420-443, October 2008 . 
[25] G. Plank, M. Liebmann, R. W. dos Santos, E. Vigmond, and G. Haase. Algebraic multigrid preconditioner for the cardiac bidomain model. IEEE Trans Biomed Eng., 54(4):585-596, 2007.

[26] G. Plank, A. Prassl, E. Hofer, and N. A. Trayanova. Evaluating intramural virtual electrodes in the myocardial wedge preparation: simulations of experimental conditions. Biophys J, 94(5):1904-1915, Mar 2008.

[27] R. Plonsey. Bioelectric sources arising in excitable fibers (ALZA lecture). Ann Biomed Eng, 16(6):519-46, 1988.

[28] N. G. Sepulveda, B. J. Roth, and J. P. Wikswo, Jr. Current injection into a twodimensional anisotropic bidomain. Biophys J, 55(5):987-99, 1989.

[29] E. Sobie, R. Susil, and L. Tung. A generalized activating function for predicting virtual electrodes in cardiac tissue. Biophys J, 73(3):1410-23, 1997.

[30] A.-K. Tornberg and B. Engquist. Numerical approximations of singular source terms in differential equations. Journal of Computational Physics, 200(2):462 - 488, 2004.

[31] N. A. Trayanova. Whole-heart modeling: applications to cardiac electrophysiology and electromechanics. Circ Res, 108(1):113-128, Jan 2011.

[32] L. Tung. A bi-domain model for describing ischemic myocardial DC potentials. $\mathrm{PhD}$ thesis, MIT, Cambridge, MA, 1978.

[33] K. H. W. J. T. Tusscher, D. Noble, P. J. Noble, and A. V. Panfilov. A model for human ventricular tissue. American Journal of Physiology - Heart and Circulatory Physiology, 286(4):H1573-H1589, 2004.

[34] F. J. van Capelle and D. Durrer. Computer simulation of arrhythmias in a network of coupled excitable elements. Circulation Research, 47(3):454-66, 1980.

[35] H. A. van der Vorst. Bi-CGSTAB: A fast and smoothly converging variant of bi-cg for the solution of nonsymmetric linear systems. SIAM J. Sci. Stat. Comput., 13:631-644, 1994.

[36] E. Vigmond, F. Vadakkumpadan, V. Gurev, H. Arevalo, M. Deo, G. Plank, and N. Trayanova. Towards predictive modelling of the electrophysiology of the heart. Exp Physiol, 94(5):563-577, May 2009. 